should be planned so that the patient receives no more protein and fat than he can digest. Carbohydrate should be given, as far as possible, as sugar, not as starch. In severe pancreatitis and in haemochromatosis, insulin therapy will be needed.

In pancreatic neoplasm the treatment is surgical.

II. Hyperthyroidism. The glycosuria is usually controlled by adequate treatment of the thyroid condition. Since these patients have a raised metabolism, it is unwise to restrict $\stackrel{0}{\circ}$ their intake of carbohydrate.

\section{Acromegaly and pituitary basophilism}

Treatment is by surgical measures when possible or by radiotherapy. The carbo- $\frac{\overrightarrow{0}}{\overrightarrow{0}}$ hydrate intake should not be unduly restricted pending treatment.

\section{Suprarenal disease}

The paroxysmal hyperglycaemia associated with the hypersecretory adenoma of the $\vec{a}$ medulla is cured if the tumour can be removed.

\title{
NERVE SUTURE
}

\section{By J. H. Кırкham, M.B., Сh.B., F.R.C.S.(Ed.)}

As there are no means yet available by which we can accelerate the regeneration of nerves after their division, it follows that the best results can be attained only by so repairing the site of division that conditions are optimum for the natural downgrowth of the axons from the proximal end to the periphery. ${ }^{1}$

It will be seen by anyone who has attempted nerve suture that the usual method of end-toend suture, by fine sutures through the sheath only, tends to cause a bunching up of the axons at the site of approximation and, in addition, a varying number of axons from both distal and proximal ends protrude through the line of anastomosis (Fig. I).

The axons which protrude from the proximal end will naturally become lost in the surrounding tissues, and can never function again. Those which protrude from the distal end will cause loss of the tubes of connective tissue and Schwann cells, by which means alone the downgrowing axons from the proximal end can attain their peripheral destinations, as the entrances to the tubes now lie outside the nerve sheath.

This loss of the possible number of axons which can ever reach the periphery must appreciably affect the perfection of the final result.
In order to obviate this, the following $\frac{7}{0}$ method has been adopted, and although the

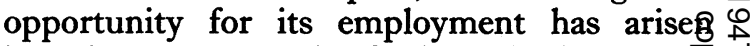
in only two cases, the final results have bee so near perfection that I consider the methoo worthy of extended trial. It may be applieđ to both primary and secondary nerve suture, although both the above cases were primary sutures.

The nerve ends having been identified, they are first trimmed square, preferably with very sharp-pointed scissors in a primary suture or a razor blade held in artery forceps in a secondary suture, as this causes minimal trauma. No instrument is used to hold the nerve itself, and the sheath is best held in fine conjunctival fixation forceps, or better still, iris forceps. The delicacy of touch required $\bigcirc$ for the operation is equivalent to that necessary for the majority of ophthalmological operations.

The distal end of the sheath is then incised $\stackrel{N}{N}$ longitudinally for about $\frac{1}{8}$ in. at points dia- N metrically opposite to each other, the two $\underset{\omega}{\mathcal{N}}$ halves then being very carefully dissected $\square$ from the underlying nerve fibres and reflected 0 distally as two cuffs of sheath. It will then be possible to cut back the nerve fibres $\frac{1}{8}$ in. further distally than the end of the sheath, thus leaving an empty tube of sheath $\frac{1}{8}$ in. 
long when the two cuffs are replaced. The position will then be as in Fig. 3 .

The proximal end is then inserted carefully into the distal tube of sheath, endeavouring as far as possible to prevent any rotation of either end. The sheath is sutured by means of fine silk on very fine curved needles (possibly the more recent fine metallic sutures, e.g., No. 35 Vitallium or Tantalum would give rise to even less reaction and fibrosis).

If this approximation is done carefully, it is possible to completely prevent bunching of the nerve fibres and, in fact, to ensure that they are end to end (Fig. 4).

It will be seen that by having the distal sheath surrounding the proximal, any proximal axon which grows peripherally cannot do otherwise than enter the distal end ; there can be no loss of axons from either end.

If the distal end, however, is appreciably smaller than the proximal, the method could be reversed, but there would be a very slight risk of the axons escaping, as would water in a pipe system in which proximal ends fit.ed round the outside of distal ends, instead of vice versa.

Although the nerve sheath is a delicate structure, it is quite possible to form a smooth anastomosis by this method, providing the sheath has not been too badly frayed during the infliction of the wound. Longitudinal splits in the sheath do not cause any difficulty as they may be used to facilitate the turning back of the cuff.

The best results with nerve suture will be obtained by primary suture in every case in which it is possible and reasonable.

Wounds which definitely exclude primary suture are grossly contaminated wounds and those in which severe intraneural damage, followed by fibrosis, is likely, such as those caused by high velocity projectiles, or by wounding with hot metal objects in which the wound is associated with burning of the tissues. Apart from these I believe that there are very few cases which will not give a better result by primary than by secondary suture. The chief reason for this is that the proliferative activity of Schwann cells, on which success of regeneration largely depends, is greatest between the 19th and 25th days, and if the nerve ends are accurately approximated before
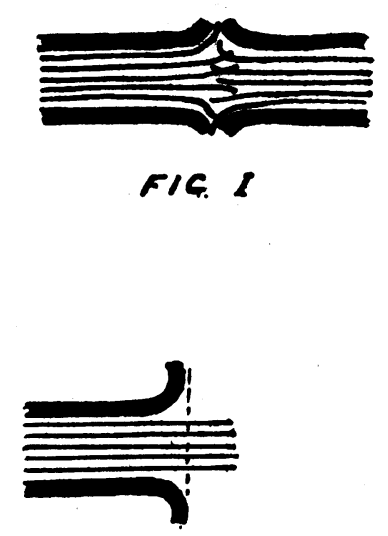

FIG. II

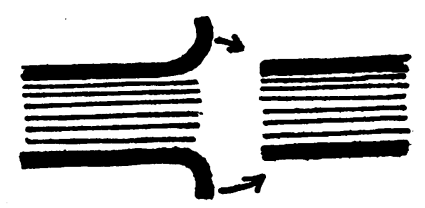

FIG. III

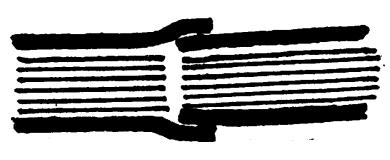

FIG. TV

this date the more likely is a successful result, as the 'soil will ripe for the seed' (the tubes being ready for the nerve fibres to grow down them). ${ }^{2}$

This is probably one of the reasons why the recovery of function is so much more perfect 'axonotmesis' than in neurotmesis; the other is that there has been little interference with the connective tissue supporting tubes down which the regenerating axons must grow in order to reach the periphery. In the present state of knowledge a neurotmesis may be 
converted into a lesion approximating as closely as possibly to axonotmesis by (a) primary suture and $(b)$ the method of suture described above, the most difficult factor involved being the estimation as to whether there is any rotation of the nerve ends.

The following points are in support of the plea for primary suture :

I. Experimentally in animals it has been proved that a "peripheral nerve injury can be primarily repaired within a grossly infected field without disturbing or delaying to any extent nerve regeneration and functional recovery.' 24

2. In most cases it is possible to bury the site of anastomosis in adjacent muscle so that there is not a continuous scar from skin to nerve which may cause dragging on the suture line. With careful excision of the wound and prophylactic chemotherapy, healing by first intention is obtained and scarring is minimal.

3. In a wound which has been carefully excised and in which prophylactic chemotherapy is employed, the risk involved in the necessary mobilization of the nerve is negligible.

4. If the nerve could possibly be ready for secondary suture by the 19th to $25^{\text {th }}$ day (when the proliferative activity of the Schwann cells is greatest) then the amount of sepsis responsible for the delay would not have affected the result of primary suture.

\section{Case Reports}

I. Male G.S., aged 8. Admitted 20/2/43. This patient fell over a tin, sustaining a laceration obliquely across the lower third of the left forearm on the ulnar side. 'T he wound was excised and sutured soon after admission. Flexor carpi ulnaris and flexor digitorum sublimis were partially severed and were repaired with catgut. The ulnar nerve was completely severed and was repaired by the method described. Prontosil powder was applied to the muscles but not the nerve.
A post-operative prophylactic course of sulphathiazole was given.

The wound healed by first intention and he $\frac{\stackrel{D}{\circ}}{2}$ was discharged from the ward on $10 / 3 / 43$; he $C$. continued to attend for massage and electrical treatment as an out-patient.

He was discharged $30 / 6 / 44$, i.e., 16 months after the accident, the result being perfect. It is almost impossible to ascertain that his ulnar nerve has ever been severed.

2. Female L.H., aged 36. Admitted $18 / 11 / 42$. This patient had a laceration of $\vec{\omega}$ the left upper arm, due to an accident on a circular saw at work. The laceration was 4-5 in. long, obliquely across the anterior and inner aspect of the left upper arm I-2 in. above the elbow joint. A portion of the biceps and the muscles arising from the anterior aspect of the internal condyle was severed. The median nerve was completely severed. Operation was performed shortly after admission. The muscles were repaired with catgut and the nerve was sutured with fine silk by the above method. Prontosil powder was applieg to the wounded muscles, but not to the nerve A prophylactic course of sulphathiazole w\$ given, the wound healed by first intention and she was discharged from the ward on $30 / 11 / 42$, continuing to attend as an outpatient for massage and electrical treatment. Treatment was discontinued on 22/9/44 when the result was almost perfect.

She was seen again on 23/1 I/45. Apart from the fact that the distal half of the index finger was slightly conical the hand and its function were indistinguishable from normal.

Objectively, no abnormality was detectable in motor function, but subjectively she com- $\bigcirc$ plained that the index finger felt a little clumsy. $D$ The latter was. also slightly colder than the other fingers and she stated that she felt pins N and needles in it during cold weather. However, these symptoms were gradually decreasing in severity. 